International Journal of Modern Physics A,

c World Scientific Publishing Company

\title{
AN INVESTIGATION INTO THE FERMILAB APPROACH TO HEAVY QUARKS ON THE LATTICE
}

\author{
ZBIGNIEW SROCZYNSKI * \\ Department of Physics, University of Illinois, 1110 West Green Street, \\ Urbana, IL 61801, USA.
}

Received

Revised

\begin{abstract}
We use a space-time asymmetric $\mathcal{O}(a)$ improved fermion action and fix the asymmetry non-perturbatively to restore the relativistic dispersion relation. We compute spectra and matrix elements of quarkonia and heavy-light mesons and compare with results obtained using a symmetric action with the Fermilab interpretation i.e. that the physics of heavy lattice quarks depends solely on their kinetic mass. We provide additional evidence to support this.
\end{abstract}

\section{The Fermilab Fermion Action}

It is known that the use of a space-time symmetric fermionic lattice action gives rise to artifacts of order $a m_{q}$ which become significant at currently accessible lattice spacings $a$ and for quark masses $m_{q}$ large enough for heavy flavour physics. This becomes manifest in the dispersion relation for lattice quarks, where a mass can be defined in two different ways; as the rest mass or the kinetic mass (denoted $M_{1}$ and $M_{2}$ respectively). On the relativistic mass shell these two definitions are equivalent but these lattice artifacts make them unequal for $a m_{q}>0$.

While there exist a number of approaches to the problems of simulating heavy quarks on the lattice, 1 the Fermilab action arises when the Symanzik improvement program is applied to the Wilson action in a mass dependent way 2 This requires that the operators in the action be allowed to be asymmetric in space and time as well as the full $a m_{q}$ dependence of their coefficients.

However, by interpreting a symmetric Wilson action in the light of the Heavy Quark Effective Theory (HQET) and normalising its operators accordingly one should be able to do without this asymmetry and use the standard SheikholeslamiWohlert (SW) action). This interpretation posits that of the two possible lattice quark mass terms, it is only the kinetic mass which is physically significant and that therefore this should be used to set the physical scale. Hitherto this has been the method used by the Fermilab and some other groups. Here, we investigate these claims in further detail.

*Current address: Theoretical Physics, University of Wuppertal, 42097 Wuppertal, Germany. 


\section{Comparison of Spectrum and Matrix Elements}

We implement the asymmetric action and adjust the asymmetry non-perturbatively until $M_{1}=M_{2}$ (within statistical errors). This has been done on a $12^{3} \times 24$ quenched lattice at $\beta=5.7$ and initial results were reported at Lattice99. 3 Since then, the non-perturbative fixing of the asymmetry has been extended from the quarkonium sector to the heavy-light sector and the statistical errors have been reduced. ${ }^{a}$ Then we use this action to compute quarkonium and heavy-light mesons with heavy quark masses corresponding to the $b$ and $c$ quark, and a light quark at the $s$ mass. We can compare our physical quantities to those obtained from previous computations on this same lattice using the SW action where $M_{1}$ is unconstrained.t
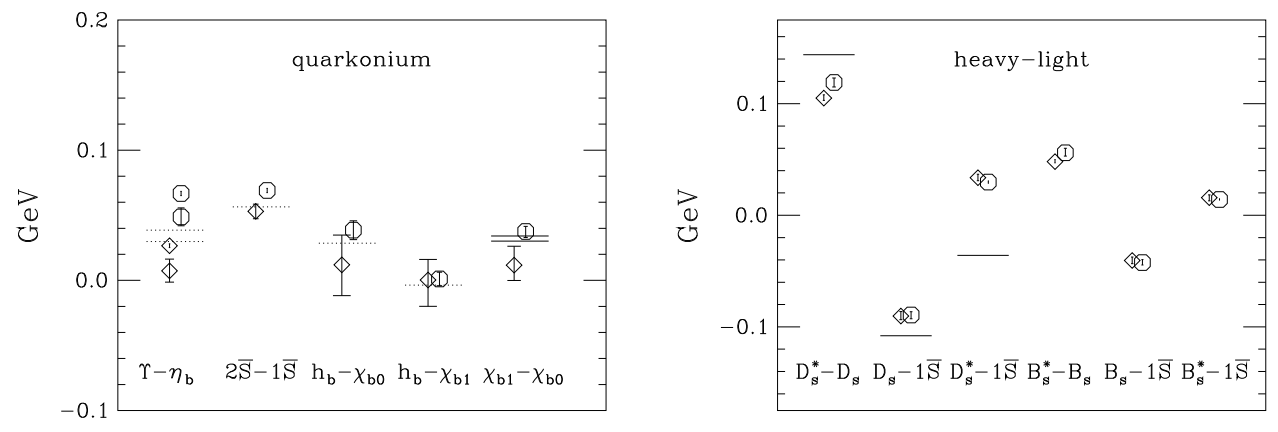

Figure 1: Comparison of results from the asymmetric $(\bigcirc)$ and the SW $(\diamond)$ actions together with experimentally measured values.

As an example, Fig. [1 shows hyperfine, radial and fine structure splittings in the $b \bar{b}$ system, and, in the heavy-light sector, the hyperfine splittings and splittings from the spin-averaged $1 \bar{S}$ state of the $c \bar{s}$ system. Physical quantities from the two actions are in good agreement despite difference in the values of $M_{1}$, and we obtain a similarly good agreement in the charmonium system and for heavy-light pseudoscalar current matrix elements,

\section{Kinetic Mass Dependence}

The asymmetry of our action allows us to arbitrarily set the ratio of $M_{1}$ and $M_{2}$, and this gives us a useful tool with which to probe their roles. We compute various mesonic physical quantities over a range of kinetic masses and either fix $M_{1}=M_{2}$ or leave it arbitrary. We add to these results those obtained using the SW action. As shown in figure 2 we find that there is clearly a smooth dependence of these various quantities on $M_{2}$.

\section{Summary}

Our results demonstrate that physical quantities depend on $M_{2}$ rather than $M_{1}$ and

a details in a forthcoming publication 

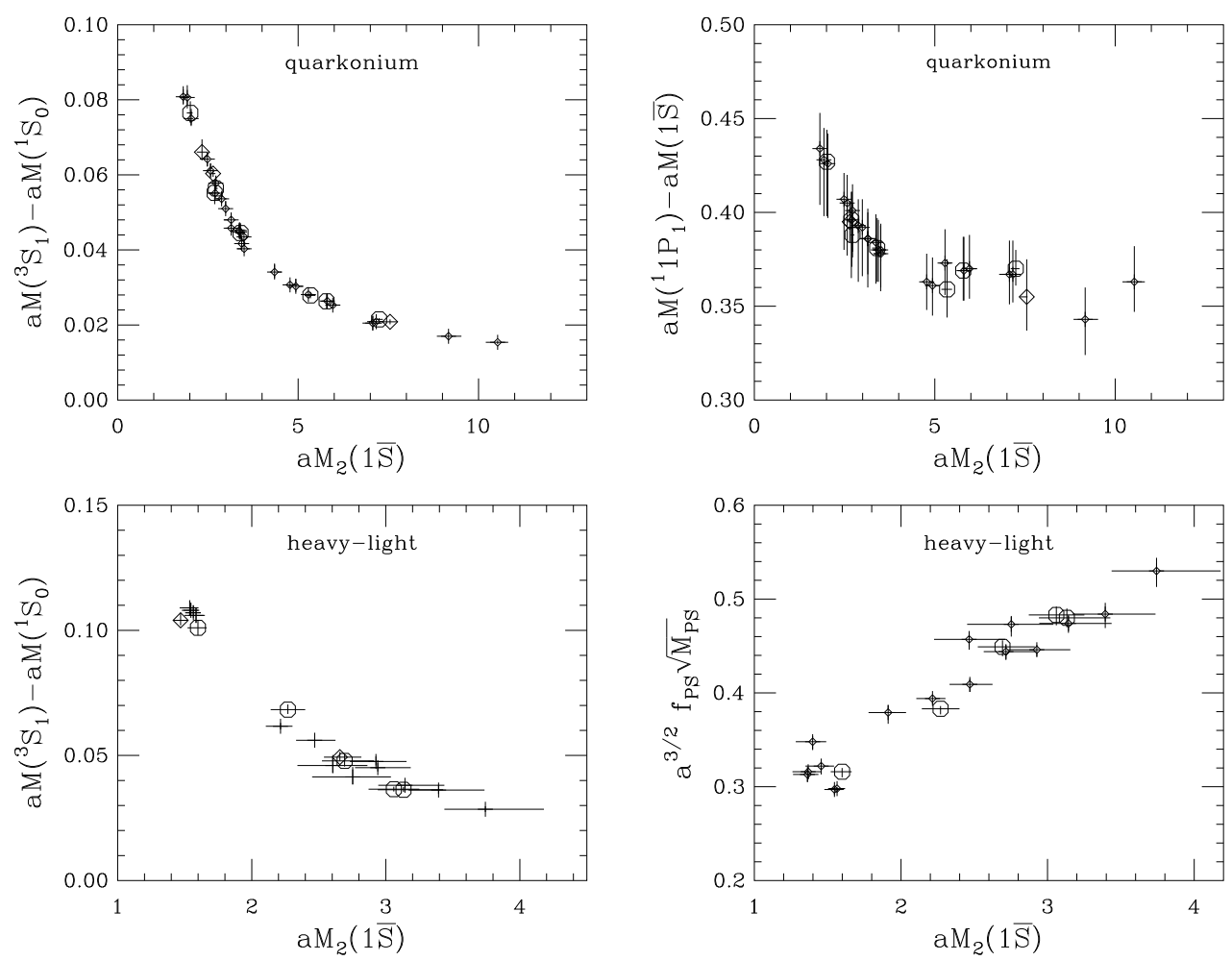

Figure 2: The dependence on $M_{2}$ of the quarkonium hyperfine and $1 P-1 S$ splittings and the heavy-light hyperfine splitting and the decay constant, using values obtained by using the non-perturbatively fixed $(\bigcirc)$, the SW $(\diamond)$ and an arbitrarily asymmetric $(+)$ action.

that the Fermilab prescription for using the SW action to compute heavy quark physics does indeed bypass the $\mathcal{O}\left(a m_{q}\right)$ artifacts that would otherwise arise.

\section{Acknowledgements}

I am grateful for the collaboration on this work of Aida El-Khadra, Andreas Kronfeld, Paul Mackenzie and Jim Simone.

\section{References}

1. T. Draper; Nucl. Phys. (Proc. Suppl.) B 73 (1999) 43-57. hep-lat/9810065

2. A. X. El-Khadra, A. S. Kronfeld and P. B. Mackenzie; Phys. Rev. D 55 (1997) 39333957, hep-lat/9604004

3. Z. Sroczynski; Nucl. Phys. (Proc. Suppl.) B 83-84 (2000) 971-793, hep-lat/9910004

4. P. B. Mackenzie, S. M. Ryan and J. N. Simone; Nucl. Phys. (Proc. Suppl.) B 63 (1998) 305-310, hep-lat/9711050

A. X. El-Khadra, A. S. Kronfeld, P. B. Mackenzie, S. M. Ryan and J. N. Simone; Phys. Rev. D 58 (1998) 014506, hep-ph/9711426 